Scientił studia, São Paulo, v. 6, n. 3, p. 409-26, 2008

\title{
ist \\ Regulación tecnológica y valores sociales: un análisis del caso farmacéutico
}

Federico VASEN

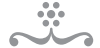

RESUMEN

El presente trabajo reflexiona sobre la interacción entre regulación tecnológica y valores sociales desde un estudio del caso farmacéutico. Partiendo de las distinciones entre riesgo material y riesgo social propuestas por Tula Molina, indaga acerca de las regulaciones vigentes sobre los medicamentos. En primer término, se analizan los valores de calidad, seguridad y eficacia, consensuados internacionalmente como parámetros técnicos para la evaluación de un fármaco. En segundo, se abordan dos cuestiones que se inscriben claramente en la discusión sobre los riesgos sociales del desarrollo tecnológico: el valor terapéutico de las innovaciones y el acceso a medicamentos esenciales. Finalmente, se propone que las regulaciones técnicas hagan mención explícita de las circunstancias sociales en las que serán implementadas.
\end{abstract}

PALABras-CLAVE $・$ Riesgo tecnológico. Evaluación de tecnologías. Regulación tecnológica.

Medicamentos. Productos farmacéuticos. Filosofía de la tecnología.

\section{INTRODUGGIÓN}

Si nos ponemos a reflexionar sobre los avances de la ciencia y la tecnología que más impacto han tenido en nuestra vida cotidiana, uno de los campos en el que su presencia es más notoria es en el de la medicina - sólo igualada quizás por los avances en las telecomunicaciones. Las nuevas terapias disponibles para mejorar los tratamientos y hacer frente a enfermedades antes intratables ubica a las ciencias de la salud como un paradigma de utilidad social de la ciencia. En este trabajo trataremos de evaluar críticamente el desarrollo de la industria farmacéutica, con el fin de mostrar que muchas veces el discurso - caro a la industria misma - del gran aporte que las farmacéuticas realizan por la salud pública no se ajusta a los hechos. Angell (2006) ha sido un gran antecedente en esta línea de trabajo, pues ha desvelado para el gran público muchos de los detalles del mercado farmacéutico que antes eran conocidos solamente en un medio más restringido. Nuestra intención es tomar algunos de estos problemas para reflexionar más ampliamente sobre el papel de las regulaciones tecnológicas tanto globalmente como también en particular en una región desfavorecida como Latinoamérica. 


\section{Medicamentos y SOGIEDAd: ¿QUÉ RIESGOS DEBEMos EVItAR GORRER?}

Para comenzar hagamos un ejercicio de imaginación y preguntémonos cuál sería el lugar de los medicamentos en una sociedad que consideramos justa, sin salirnos de los límites del capitalismo de mercado actual. Creo que podríamos acordar en alguno de los siguientes puntos:

(1) Los medicamentos están disponibles a precios accesibles y/o cubiertos por algún tipo de sistema de salud.

(2) La inversión de las empresas productoras se concentra en las actividades de investigación y desarrollo de nuevos productos.

(3) Los nuevos medicamentos que salen al mercado representan una ventaja frente a los que ya se vendían, sea en términos terapéuticos o económicos.

(4) La investigación se guiará prioritariamente a las enfermedades que tengan mayor tasa de mortalidad y afecten a mayor número de personas.

(5) Los médicos recetan medicamentos de acuerdo a información científica de calidad.

(6) Todos los medicamentos disponibles en el mercado cumplen con criterios de calidad, seguridad y eficacia.

(7) Las innovaciones pueden ser protegidas con títulos de propiedad intelectual que garanticen una renta monopólica por un plazo de tiempo determinado a fin de recuperar la inversión original, sin que esto conlleve niveles de precios que los vuelvan inaccesibles. Pasado el plazo, se permitirá una competencia abierta.

Sin embargo, la realidad nos muestra su costado más duro cuando confirmamos que ninguna de estas afirmaciones es verdadera, sino muchas veces más bien la opuesta:

( '’) De no mediar intervenciones humanitarias o de una regulación o de la provisión estatal, el precio actual de muchos medicamentos los vuelve inaccesibles a grandes poblaciones que los necesitan. Quick et al. (2002) señalan que hoy un tercio de la población mundial - unos dos mil millones de personas - no tienen acceso a medicamentos considerados esenciales.

(2') La inversión de las empresas farmacéuticas que se dicen innovadoras se concentra en mayor medida en actividades de marketing y promoción antes que en investigación y desarrollo de nuevos productos (cf. Angell, 2006, Cap. 1-3).

(3') Las nuevas drogas que salen al mercado no constituyen en su mayoría una mejora terapéutica frente a otras ya existentes, si bien las empresas productoras las promocionan como tales (cf. Garattini, 1997; Ugalde \& Homedes, 2006a; Angell, 2006, Cap. 5). 
(4') El gran número de nuevos productos comercializados se concentra en enfermedades crónicas que afectan principalmente a pacientes en países desarrollados como la hipercolesterolemia o la hipertensión. Sólo el 1\% de 1223 nuevos fármacos lanzados al mercado entre 1975 y 1997 fueron destinados a enfermedades tropicales transmisibles (cf. Trouiller et al., 2001).

(5') Los médicos están altamente influidos por las empresas farmacéuticas, que financian viajes y dan premios por su conducta prescriptiva. Las empresas también realizan capacitaciones para los médicos con un formato académico y gestionan la publicación de artículos positivos en revistas científicas de alto impacto (cf. Wazana, 2000; Sismondo, 2007).

(6') Existen medicamentos falsificados de muy baja calidad en el mercado que pueden ser tóxicos (cf. Newton et al., 2002). Por otra parte, los requisitos de las agencias regulatorias para los estudios de seguridad y eficacia son cada vez menores (cf. Abraham \& Reed, 2002).

( $\left.\eta^{\prime}\right)$ El sistema de protección de las innovaciones por medio de patentes se ha vuelto extorsivo y no cumple su rol de estimular la innovación, en tanto se admiten patentes por productos que no representan una verdadera novedad y se justifican los altos precios en función de estas actividades de Investigación+Desarrollo (I+D). La industria utiliza cualquier argumento o laguna legal para extender su monopolio y evitar la competencia (cf. Angell, 2006, Cap. 10).

Un breve repaso del primer grupo de afirmaciones permitirá distinguir dos tipos de valores que las fundamentan como escenario deseable. En primer término, las ventajas terapéuticas mencionadas en el punto (4) y los atributos de calidad, seguridad y eficacia presentes en el punto (5) nos remiten a la calidad de la investigación científica que fundamenta un nuevo fármaco. Es decir, se espera que el nuevo producto actúe eficazmente sobre la enfermedad y que lo haga sin ser nocivo en otros aspectos sobre el paciente, esto es, que sea seguro. Por otra parte, el proceso de producción debe cumplir con normas de calidad que permitan cumplir adecuadamente su función (que contenga la cantidad de principio activo que declara, por ejemplo). La garantía de estos valores estaría dada por los estudios científicos que avalan las propiedades del fármaco, tanto en términos absolutos como en comparación con los otros fármacos ya existentes para la misma condición. Podemos suponer que una evaluación que se diga objetiva de estas propiedades puede llevarse a cabo de acuerdo a la metodología científica característica de la ciencia médica, tanto en una primera etapa en animales como luego en estudios clínicos. Este tipo de evaluaciones nos retrotrae al ideal clásico de la ciencia moderna como conocimiento y control de los fenómenos naturales y de evaluación de los resultados de nuestra intervención en ellos. 
Si observamos las afirmaciones realizadas queda claro que si nos guiamos por los estándares de la ciencia para evaluar los productos farmacéuticos no podremos llegar mucho más allá de afirmar que deben probar su eficacia y seguridad mediante cierto tipo de estudios, o ser fabricados en un rango determinado de especificaciones. El resto de lo afirmado en (1)-(7) no puede explicarse en estos términos. Al reflexionar sobre estos puntos resulta evidente que el medicamento no es un simple artefacto tecnológico que puede cumplir una función de acuerdo a un diseño basado en ciertos conocimientos científicos y habilidades técnicas. Para ellos es necesario otro tipo de justificación. La más sólida está basada en el derecho a la salud, uno de los más aceptados universalmente. Tipificado como Derecho Humano en el art. 14 del Pacto Internacional de Derechos Económicos, Sociales y Culturales de 1966, en su interpretación clásica, incluye el acceso a medicamentos considerados esenciales (cf. Alto Comisionado de la ONU para los DDHH, 1966, 2000). En este marco normativo, podemos dar una mejor justificación a afirmaciones como (1) y comprender el espíritu de sostener (2), (3), o (4). Sin embargo, no son suficientes para justificarlas en detalle, pues el derecho a la salud nada afirma sobre el rumbo de la investigación biomédica o farmacéutica ni sobre el valor terapéutico de las innovaciones, sino que se limita a garantizar el acceso universal a ciertos medicamentos, más allá de muchas otras garantías que exceden lo farmacéutico.

Si observamos los puntos (1')-( $\left.\eta^{\prime}\right)$ nos damos rápidamente cuenta de que si se quieren garantizar estándares mínimos de calidad, seguridad y eficacia así como de acceso a la salud no podemos descansar en la regulación misma de las compañías o del mercado. Esto nos obligaría a prever riesgos de dos tipos distintos, siguiendo la clasificación que Tula Molina (2006) propone para el desarrollo tecnológico en general: un riesgo material y un riesgo social. Según el primero, sólo hay responsabilidad en el caso de que algo falle en el diseño tecnológico. En el segundo de los casos, existe también responsabilidad en el caso en que nada falle en el diseño, reconociendo que los problemas éticos y sociales vinculados a la ciencia están relacionados con el respeto a los valores mantenidos por la sociedad. En el caso puntual del desarrollo farmacéutico podemos decir que el riesgo material estaría dado por el cumplimiento de los estándares de calidad, seguridad y eficacia referidos anteriormente como (6). Sin embargo el cumplimiento de estas pautas es necesario aunque no suficiente para evitar el riesgo en sentido social, que abarcaría muchos más aspectos, como ser el acceso a medicamentos esenciales o la discusión sobre el direccionamiento de las actividades de I+D financiadas en buena medida por fondos privados.

En este trabajo nos focalizaremos en tres aspectos. En primer término, resumiremos en qué consisten los criterios en los que se basa la evaluación de los nuevos productos farmacéuticos, tanto los referidos al proceso de elaboración industrial de 
un preparado como a las características del principio farmacológicamente activo. De este modo trataremos de ejemplificar lo que se hace para evitar el riesgo en sentido tecnológico estrecho, lo que hemos llamado riesgo material. Luego analizaremos dos puntos relacionados con el riesgo en sentido más amplio: el problema de la "calidad" o valor terapéutico de los medicamentos innovadores y la experiencia de la redacción de la lista de medicamentos esenciales de la OMS (tendiente a ampliar el acceso a medicamentos en países subdesarrollados). A través de ellos podremos reflexionar acerca de la relación entre regulaciones tecnológicas y valores sociales.

\section{Controlando El RIESGo MATERIAL: GALIDAD, SEgURIDAD Y EFICACiA EN LA EVALUAGIÓN DE PRODUGTOS FARMAGÉUTIGOS}

Los medicamentos reúnen junto con muchos otros productos tecnológicos una serie de características: no serían posibles sin los resultados de la investigación científica, fueron pensados para ser utilizados de una forma determinada (poseen un "manual de instrucciones" muy específico: el prospecto), son producidos en escala industrial etc. En síntesis: son resultado de una detallada acción de diseño. ${ }^{\mathbf{S}}$ Sin embargo, tanto su mecanismo de acción como su función social los hacen pasibles de un control ex ante mucho más estricto que lo que podría suceder con otros artefactos. ${ }^{2}$ La importancia de estos controles se vio potenciada por algunos casos emblemáticos como el de la talidomida, una suerte de Chernobyl para la industria farmacéutica. Muchos recordarán los efectos nefastos sobre los fetos de esta droga administrada para las náuseas a mujeres durante los primeros tres meses de embarazo en la década de 1950 y 1960.

En función de evitar peligros derivados de una falta de estudios sistemáticos previos a la comercialización, en el plano institucional se instauró la idea de la necesidad de agencias de regulación de medicamentos. La FDA, creada mucho antes, tomó el tema en los EEUU, en América Latina la ANMAT argentina, creada recién en 1992, fue la pionera. Sin embargo, existen países en nuestra región como Uruguay o Paraguay que aun hoy no cuentan con una agencia de este tipo (estos temas son tratados por reparticiones dentro de los ministerios de salud). En el plano técnico comenzó a priorizarse

\footnotetext{
1 Broncano (2000) propone la existencia de un diseño que puede ser estudiado y corregido sin necesariamente ser ejecutado como el criterio de demarcación entre técnicas artesanales y tecnologías. En el caso de los medicamentos puede pensarse en dos niveles diferentes: el diseño y la síntesis de la molécula del principio activo, y el diseño de cada preparado farmacéutico en particular, junto con los excipientes, el envasado y el rotulado.

2 El potencial cancerígeno de los teléfonos celulares, por ejemplo, se discute sólo tangencialmente y mucho tiempo luego de su comercialización.
} 
la redacción de normativa sobre los controles que los medicamentos debían pasar para que fuera autorizada su comercialización. Los principios consagrados por la OMS se centran en tres conceptos: calidad, seguridad y eficacia. ${ }^{3}$

La calidad se relaciona con el proceso mediante el cual se produce el fármaco, se evalúa entre muchas otras cosas la calidad del principio y los excipientes y las características del proceso de elaboración. Las normas de calidad están tipificadas en un documento denominado Buenas Prácticas de Fabricación y Control, conocido generalmente como GMP por sus siglas en inglés. La OMS genera periódicamente documentos con recomendaciones pero queda en jurisdicción de cada país adoptar las normas de GMP que logre consensuar localmente, manteniendo un equilibrio entre la capacidad de sus industrias y los requerimientos de la salud pública. Las agencias regulatorias realizan inspecciones a las plantas elaboradoras y emiten certificados de cumplimiento de GMP para las distintas líneas de producción. Como ejemplo de problemas de calidad, podemos recordar el famoso caso de la intoxicación con dietilenglicol en la fabricación de propóleo en Argentina que dio lugar a la creación de la ANMAT en 1992 y el caso similar que se observó en Panamá con un jarabe infantil en 2006.

La eficacia, en cambio, se centra no tanto en cada preparación específica sino en el principio activo mismo (generalmente una droga de síntesis), en su capacidad para cumplir la acción farmacológica que se le atribuye. Esta se mide a través tanto de los ensayos preclínicos in vitro e in vivo (ratas, ratones, perros) como de los ensayos clínicos realizados previamente a su autorización. Tanto los primeros como los segundos deben adecuarse a normas estandarizadas: las Buenas Prácticas de Laboratorio (GLP) y las Buenas Prácticas Clínicas (GCP), que incluyen también las salvaguardas bioéticas para la investigación en seres humanos expresadas en la Declaración de Helsinki y los posteriores normas de la OMS. Cabe aclarar que las agencias regulatorias analizan la eficacia de una droga en términos absolutos y no relativos, es decir, evalúan si es mejor que un placebo y no si es mejor que los demás productos en el mercado. Esto traerá consecuencias luego para lo que abordaremos en la sección siguiente.

Por último, la seguridad se relaciona con los efectos no deseados del medicamento. Un porcentaje de los efectos adversos se descubre durante la fase de ensayos previos a la comercialización, mientras otro porcentaje recién puede detectarse una vez que el medicamento ya salió a la venta y es administrado a gran número de personas. En esta etapa entra en juego la farmacovigilancia, un sistema mundial de vigilancia sanitaria que recibe reportes de efectos adversos no deseados en todo el mundo y

3 Información actualizada del área encargada de estos temas en la OMS puede encontrarse en <http://www.who.int/ medicines/areas/quality_safety/en/>. 
los analiza estadísticamente, estimando el nivel de gravedad y la frecuencia de su aparición. Cada país tiene su propio sistema de farmacovigilancia, que recopila los datos a nivel nacional y luego son centralizados en un centro colaborador de la OMS. En base a estos análisis pueden decidirse medidas de intervención tales como retiros de productos del mercado, cambios en la condición de venta y modificación en los prospectos, lo cual entraña no sólo una dificultosa cuestión de análisis y estimación de los riesgos, sino también de comunicación pública de los mismos. Como caso de retiro del mercado, puede citarse el conocido producto Vioxx ${ }^{\circledR}$ (rofecoxib) de Merck, Sharpe \& Dohme. Estudios científicos confirmaron que el grupo de antiinflamatorios inhibidores de la ciclooxigenasa-2, al que pertenecía producía un aumento del riesgo cardíaco. Un caso reconocido de cambio en la información del prospecto fue el del Viagra ${ }^{\circledR}$ (sildenafil), contraindicándose para pacientes con condiciones cardíacas previas. Por último, un caso extremadamente complejo es el del Cytotec ${ }^{\circledR}$ (misoprostol), un fármaco para combatir la úlcera gástrica cuya capacidad para provocar abortos ha hecho que su venta sea mucho más restringida en los países donde esta práctica se encuentra prohibida.

Las normas o principios básicos de calidad, seguridad y eficacia se revisan continuamente. Para el caso de la calidad, la adopción de controles más o menos estrictos depende en buena medida de las capacidades de la industria local. Los requerimientos de seguridad y eficacia se discuten en los países desarrollados con más intensidad que en los subdesarrollados, ${ }^{4}$ pues estos últimos muchas veces "confían" en las autoridades regulatorias de los países centrales para la evaluación de los datos de seguridad y eficacia. Este es el caso de Argentina, donde la norma que regula el registro de medicamentos (Decreto 150/92) instaura un principio de similaridad, según el cual no es necesario analizar la información de seguridad y eficacia de un principio activo (en una concentración, indicación y forma farmacéutica específica) que ya se comercializa en alguno de los países llamados "de alta vigilancia sanitaria”. Estos datos sólo se evalúan en caso de que el producto sea considerado una "novedad terapéutica”, esto es, no sea comercializado en ninguno de estos países, situación harto excepcional.

4 A instancias de la industria farmacéutica, EEUU, la Unión Europea y Japón han entrado en un proceso de armonización de la regulación farmacéutica con el objetivo de que la información sobre calidad, seguridad y eficacia presentada para registrar un producto en un país sea igualmente válida en el otro (a través de las guías de la International Conference on Harmonization - ICH). A nivel panamericano, la OPS ha creado una Red para la Armonización de la Regulación Farmacéutica (PARF/PANDRH) que persigue en última instancia ese mismo fin. En nuestra región, permite "nivelar para arriba" las regulaciones de los países más rezagados. Sin embargo, también se corre el riesgo de que armonizar requisitos muy elevados deje fuera del juego a las industrias locales. 


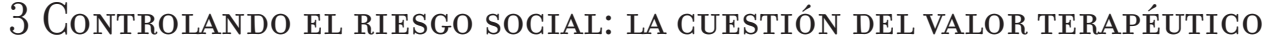 DE LAS INNOVAGIONES Y EL AGGESO A MEDICAMENTOS ESENGIALES}

El consenso acerca de las medidas político-regulatorias a tomar sobre el campo farmacéutico desaparece rápidamente cuando pasamos del plano de lo que hemos denominado "riesgo material" al más amplio del "riesgo social". 5 No existe una respuesta unificada a los problemas sobre el acceso, la influencia de la industria en las prácticas prescriptivas de los médicos, la aparición de nuevos fármacos de escaso valor terapéutico o la falta de innovaciones para enfermedades endémicas del tercer mundo. En este trabajo presentaremos el estado de la cuestión en dos de estas problemáticas: el valor terapéutico de las innovaciones y el acceso a medicamentos esenciales, para luego intentar extraer algunas conclusiones más abarcativas sobre la interacción entre regulación y valores sociales en el desarrollo tecnológico.

\subsection{INNOVACIÓN Y MEDICAMENTOS me too: ENTRE GURAR Y VENDER}

El cambio tecnológico suele considerarse en dos niveles. Por una parte puede hablarse de mejoras a un proceso ya existente, que tiendan a optimizar sus resultados, adaptando, reemplazando o renovando parte del producto o proceso en cuestión. Más allá de esto la historia de la tecnología muestra también grandes innovaciones que producen un "gap", un salto tecnológico cualitativo frente a lo ya existente. En el caso particular de la industria farmacéutica esta distinción se vuelve central, tiñéndose de fuertes consecuencias éticas. Como señalamos al comienzo del trabajo - en los puntos (3’) y (4') buena parte de los nuevos medicamentos en salir al mercado no representan una mejora terapéutica sustancial respecto de otros ya disponibles. A estos últimos se los denomina medicamentos me too (“yo-también”), en función de que aspiran a una porción de mercado cuya rentabilidad ya fue demostrada por el primer medicamento innovador del grupo. Más puntualmente, se trata de moléculas con pequeñas modificaciones con respecto a las ya disponibles y que no siempre presentan una avance terapéutico, y en la mayoría de los casos en que sí lo hacen no suele pasar más que por mejoras menores como por ejemplo en la forma de administración (deben tomarse cada 12 en vez de cada 6 u 8 horas; cf. Angell, 2006, p. 97-9). Si bien mantienen un mecanismo de acción similar, al tener una estructura química diferente, no están su-

5 No queremos señalar con esto que existe un consenso universal sobre los estándares técnicos aceptables, pero no parece haber discusión sobre la naturaleza de los estándares mismos. Puede discutirse el tipo de estudios necesarios para estudiar la seguridad, pero nadie duda de que la seguridad es un valor que debe ser resguardado por toda la comunidad farmacéutica y especialmente por los Estados. 
jetas a la patente del producto innovador y pueden ser patentadas por otro laboratorio. Ejemplo de esto puede ser la trayectoria del grupo de los antiinflamatorios no esteroides (AINE) ya mencionada a propósito del Vioxx. A los grupos ya existentes de salicilatos (aspirina), derivados del ácido propiónico (ibuprofeno, naproxeno), oxicams (piroxicam, meloxicam) y ácidos fenilacéticos (diclofenac), se agregan en 1999 los inhibidores selectivos de la ciclooxigenasa-2, produciendo una verdadera revolución en el grupo, pues disminuían considerablemente los efectos adversos en el tracto gastrointestinal característicos de los AINE tradicionales (cf. Green, 2001). Tras la aparición de los dos primeros principios activos del grupo (Vioxx-rofecoxib-MSD, Celebrex-celecoxib-Pfizer) pronto surgieron otros que buscaron ocupar ese espacio (Bextra-valdecoxib-Searle, Dynastat-parecoxib-Pfizer, Arcoxia-etoricoxib-MSD, Prexige-lumiracoxib-Novartis), muchos de los cuales fueron retirados del mercado en 2005 luego de la comprobación del aumento del riesgo cardíaco por el rofecoxib.

La industria farmacéutica suele presentarse a sí misma tan bienintencionada como innovadora, como un sector que invierte grandes sumas en el desarrollo de nuevos productos que serán capaces de salvar muchas vidas, y ante cualquier intento de regular sus prácticas o sus ganancias, reaccionan resaltando lo que sucedería si no hubiera innovación en absoluto. ${ }^{6}$ La realidad muestra que este discurso no es cierto, la presencia de medicamentos me too en el mercado es muy importante y no responde a la innovación para salvar vidas sino a una innovación parcial (con una cuota de imitación) para ganar mercado. Garattini (1997), tras un estudio acerca de los 244 nuevos principios activos comercializados en Italia entre 1984 y 1992, concluye que sólo el 9\% de los representan mejoras sustanciales, frente a 24,1\% de me too con alguna ventaja y $4,2 \%$ de me too sin ventajas. Angell analiza el mercado norteamericano y saca conclusiones igualmente preocupantes: en 2001 y 2002, se aprobaron en EEUU únicamente 7 nuevos principios activos que representan una mejora sustancial cada año, cifra que está en franco descenso, sólo en 1998 fueron 16 (cf. Angell, 2006, p. 77).

La ventaja de invertir en I+D de moléculas me too se entiende claramente en una lógica económica, es simplemente una inversión de menor riesgo. Ahora bien, ¿hay una justificación desde el punto de vista de la salud pública? Uno puede preguntarse hasta qué punto es beneficiosa toda la inversión en productos me too cuyas ventajas terapéuticas son bajas o nulas (pero ampliamente resaltadas en los folletos de mercado de los laboratorios, cf. Sismondo, 2007), en vez de dedicarse a la investigación de nuevos grupos moleculares. Lee (2004), en un artículo en el respetado New England

6 Cuesta creer que cualquier cambio en la regulación podría representar una pérdida económica tan grande que peligre la viabilidad del negocio. Según la revista Fortune, entre 1991 y 2001, la industria farmacéutica fue la más rentable de los EEUU, cinco veces y media por encima del promedio (cf. Ugalde \& Homedes, 2006a). 
Journal of Medicine, defiende la existencia de este tipo de productos argumentando razones económicas que repercuten positivamente en la salud pública. Según este autor, la existencia de los me too favorece la competencia y permite que los precios bajen. Afirma que llega un punto en que los me too dejan de ser beneficiosos terapéuticamente - es interesante este reconocimiento por alguien que defiende el punto de vista de la industria - y sólo pueden competir bajando el costo, pues los médicos ya se han acostumbrado a recetar el primer producto del grupo en salir a la venta, el verdaderamente original. Intenta demostrar esto con un pequeño estudio empírico de precios en los EEUU (toma únicamente cinco grupos y dos productos por grupo), que permitiría concluir que los me too son más baratos y justificar así su existencia. Además, la presión de que el mercado al que aspiran ya esté cubierto obliga a pensar en otras estrategias además del costo para mejorar los productos ("maketing inteligente" en palabras de Lee). 7 En el mercado farmacéutico, el costo no es tan importante como en otros mercados, en tanto quien paga el medicamento no es quien lo elige, y quien lo elige no tiene en cuenta su costo. Así se entienden las grandes inversiones en promoción y mercado, pues como señala Angell si el producto es realmente mejor que el anterior, no es necesario gastar mucho en publicidad, los médicos pueden reconocer claramente la diferencia. La perspectiva de Lee encubre que no todos los mercados funcionan como el norteamericano (en Argentina esa comparación sería muy difícil de realizar en función de los medicamentos similares) y, en segundo término, iguala engañosamente la innovación en pequeños puntos con la innovación a gran escala al afirmar que "los productos me too no significan que la imitación ha reemplazado la innovación en la investigación farmacéutica" (Lee, 2004, p. 212).

Una segunda perspectiva - más allá de lo económico - según la cual podemos analizar esto es la de los filósofos de la tecnología como Quintanilla (1988) y Broncano $(2000,2007)$. Desde la perspectiva de estos autores, creo que podríamos entender la aparición de los me too como un proceso de optimización y aumento de la eficiencia, del control intencional del diseño, connatural a la acción técnica misma. De hecho, un producto me too tiene un piso de eficiencia mucho más alto que un primer producto innovador, pues descansa en la experiencia del primer fármaco del grupo. Sin embargo, para discutir esto Broncano nos acerca también la distinción entre la dimensión objetiva de las posibilidades y la dimensión subjetiva de las oportunidades (Broncano, 2000 , p. 130). De todas las posibilidades pragmáticas que nos presenta el desarrollo tecnológico no todas son legítimas, y si bien algunas son legítimas de por sí, quizás no

7 En buena medida se trata de convencer a los médicos a través de múltiples beneficios (desde viajes hasta su nombre en artículos académicos en revistas de primera línea), de que el producto me too es mejor (cf. Wazana, 200o; Sismondo, 2007; Angell, 2006, Cap. 7-9). 
sea legítimo emprenderlas considerando un marco social más complejo que relativiza su urgencia. El que los me too sean percibidos como oportunidades no está relacionado tanto con la lógica de la salud pública como por la lógica comercial de desarrollar un producto cuyo mercado está ya asegurado. A ello ayuda la lógica misma del cambio técnico que suele trabajar con pequeñas innovaciones. Por tanto, si bien es cierto que es natural, y no de por sí criticable, que existan pequeñas optimizaciones de productos (no es lo mismo una lectora de CD 4x que 52x), el mercado farmacéutico tiene características particulares por su función primordial en la salud pública y es en relación con esta función que deben priorizarse las nuevas líneas de investigación. Ugalde y Homedes encuentran la causa de estos males directamente en el capitalismo:

el capitalismo ha forzado a la industria farmacéutica a invertir una gran cantidad de fondos de I+D a producir drogas que no añaden valor terapéutico al mercado. El propósito de estas drogas es competir con las drogas innovadoras exitosas [blockbuster], es decir, aquellas que generan más de mil millones de dólares anuales en ventas. Es esta competencia para ganar una porción del mercado la que obliga a la industria a realizar grandes inversiones en promoción y mercado. Aquellos que fallan en la competencia son comprados por otros. Los partidarios del capitalismo sostienen que la competencia fomenta la innovación, pero éste no parece ser el caso para la industria farmacéutica (Ugalde \& Homedes, 2006a, p. 29).

Este patrón de desarrollo innovativo en la industria farmacéutica lleva a que las enfermedades endémicas del tercer mundo queden doblemente relegadas, pues si el dinero no se invierte en me too, la prioridad son las terapias realmente innovadoras para las enfermedades más rentables (crónicas de los países desarrollados), recién en tercer término podrían aparecer medicamentos para enfermedades desatendidas. La urgencia de pensar estrategias para fomentar su desarrollo salta a la vista cuando tomamos en cuenta que, como ya se mencionó, menos del 1\% de los 1223 nuevos fármacos lanzados al mercado entre 1975 y 1997 fueron destinados específicamente a las enfermedades tropicales transmisibles (cf. Trouiller et al., 2001). ${ }^{\mathbf{8}}$

8 Las políticas de medicamentos huérfanos - facilitar los registros y extender las patentes para enfermedades poco usuales - se han focalizado más en enfermedades raras en países desarrollados antes que en enfermedades endémicas en los subdesarrollados (Villa et al., 2008 proponen trabajar en esta línea para enfermedades desatendidas). 


\subsection{FaGilitando eL AGGeso: LA INIGiativa DE MEdicamentos ESENGIALES DE LA OMS}

Habíamos señalado anteriormente que podíamos pensar dos situaciones diferentes en las que el riesgo social se manifestara. Por una parte, si no existen medicamentos apropiados para paliar las enfermedades que se cobran más vidas a edades más tempranas, debemos encontrar la forma de redireccionar la investigación y el desarrollo farmacéutico a estos objetivos. Respecto a esto, analizamos la situación de los me too y de la tendencia de nuevos productos que predominan en la industria. El segundo tema que abordaremos se relaciona con la accesibilidad de los medicamentos ya existentes. Para tratar gran cantidad de enfermedades mortales en los países subdesarrollados no se requieren nuevos fármacos. La mayoría está ya disponible, pero las condiciones políticas, económicas y de salud pública no permiten que llegue efectivamente a aquellos que los necesitan. Las estadísticas de la OMS son sin embargo optimistas: calculan que hoy aproximadamente dos tercios de la población mundial tiene acceso a los fármacos básicos, mientras que en 1977 era menos de la mitad (cf. Quick et al., 2002). Una de las causas de esta tendencia favorable se debe a la política de medicamentos esenciales que comentaremos a continuación.

Con el propósito de promover el acceso a los medicamentos en las situaciones socioeconómicas más complejas, la OMS decidió en 1975 introducir los conceptos de "medicamentos esenciales" y "política nacional de medicamentos". La idea que está a la base del concepto de medicamentos esenciales es que de todos los principios activos disponibles, algunos son más "esenciales" que otros, i.e. menos prescindibles para la atención primaria de la salud. La necesidad de una cuidadosa selección vuelve a llamar la atención sobre lo que habíamos comentado anteriormente: la existencia de muchos fármacos cuya innovación en términos terapéuticos es dudosa o nula.

La definición de medicamento esencial de la OMS que transcribimos a continuación señala tres criterios básicos:

Los medicamentos esenciales son aquellos que satisfacen las necesidades sanitarias prioritarias de la población. Se seleccionan de acuerdo a la prevalencia de la enfermedad, la evidencia sobre su seguridad y eficacia y la relación comparativa costo-efectividad. Se tiene la intención de que estén disponibles en el contexto de sistemas de salud en funcionamiento, en todo momento, en las cantidades y formas farmacéuticas adecuadas, con una calidad asegurada y a un precio accesible para el individuo (Hogerzeil, 2006, p.371-2).

Lo interesante de esta definición es que involucra aspectos tanto técnicos como sociales. El énfasis en la seguridad y la eficacia nos retrotrae a aspectos que considera- 
mos técnicos del medicamento, que son habitualmente juzgados por las autoridades regulatorias como condición necesaria para la comercialización del fármaco (parte del "riesgo material"). La prevalencia de las enfermedades y la relación costo-beneficio exceden la evaluación técnica y se relacionan con el medio social particular en el que la lista se aplicará. Por eso, si bien la OMS hace una primera lista de medicamentos esenciales, son los países los que en última instancia deciden qué medicamentos quedan incluidos.

El proceso de selección de los medicamentos es complejo. El comité que decide está formado por doce expertos de diferentes áreas geográficas y con experiencia en los siguientes temas: farmacología clínica, clínica médica, desarrollo de directivas, métodos sistemáticos de búsqueda bibliográfica, análisis de riesgo y de costo-beneficio. El comité se reúne cada dos años, durante los cuales se va conformando la agenda de cada reunión. Los pedidos de inclusión o modificación por parte de la industria, las universidades o las asociaciones de pacientes deben ser realizados a través del departamento de la OMS correspondiente para la enfermedad tratada. Los representantes de estos grupos de interés no pueden asistir a las sesiones del comité en las cuales se toman decisiones, aunque sí pueden hacerlo a una primera sesión abierta en la cual tienen un espacio para expresar sus puntos de vista a los miembros del comité (cf. OMS, 2001, 2007). Por otra parte, los mismos miembros del comité deben hacer público si tienen algún conflicto de interés, absteniéndose de participar en las discusiones en las que podrían aportar algún sesgo (tener un pariente trabajando para una empresa farmacéutica o haber recibido financiamiento de ella para un viaje es causal suficiente para abstenerse de discutir temas que involucren sus productos).

Con respecto a las aplicaciones de esta lista, la OMS pretende que se transforme en un instrumento de política sanitaria, que permita decidir qué medicamentos distribuir en los programas sanitarios que llevan adelante principalmente los países subdesarrollados. La lista les permite a estos países saber cuál es el menú básico de medicamentos que cumplen con especificaciones básicas y se han mostrado más seguros a lo largo del tiempo. Además les da una poderosa herramienta para luchar contra los ubicuos agentes de lobby de las empresas farmacéuticas, que quieren imponer a cualquier precio su producto frente a los competidores y convencer a los responsables políticos de que se trata del más adecuado.

El documento de OPS Los medicamentos esenciales en las Américas: logros, dificultades y retos (2007) permite tener un panorama general de las aplicaciones concretas que ha tenido la lista de medicamentos esenciales en América Latina. En la Argentina el ejemplo más relevante es el del programa Remediar del Ministerio de Salud de la Nación, que distribuye gratuitamente un botiquín de medicamentos a los centros primarios de atención de la salud. La selección de los medicamentos presentes en el boti- 
quín fue realizada de acuerdo a los criterios de la lista de OMS (OPS, 2007; Ugalde \& Homedes, 2006b). En un trabajo sobre utilidad social del conocimiento científico para la formulación de políticas, Estébanez (2007) trae a colación la experiencia del Plan Remediar como un caso en el que la autoridad científica permite frenar las acusaciones de que la selección de los principios activos a incluir en el listado pudo haber estado sesgada por los intereses comerciales de las empresas. De este modo también los funcionarios públicos ven con ojos positivos la utilización de conocimiento científico para la formulación de políticas.

\section{REFLeXiones finAles}

Al distinguir entre riesgo material y riesgo social - al comienzo del trabajo - señalamos que en el desarrollo tecnológico hay mucho más en juego que un control técnico de que "las cosas no fallen". La evaluación que se haga de cada artefacto tecnológico, en tanto es parte de una realidad social compleja que incluye factores que van más allá de lo técnico - económicos, éticos, políticos-, debe ser necesariamente multidimensional.

En esta oportunidad nos hemos referidos a un artefacto tecnológico de gran valor social: los medicamentos. Insumo imprescindible para alcanzar los estándares mínimos de salud pública, los productos farmacéuticos están más claramente sujetos al control. Este control no es ya un control meramente técnico, si bien éste claramente existe (como hemos revistado en la sección 3) y es necesario. Siguiendo las distinciones de Molina (2006), puede hablarse también de control en un sentido más amplio para todas aquellas cuestiones relacionadas con los medicamentos cuando evaluamos su función social y no su función técnica. Esta segunda noción de control no se relaciona principalmente con el dominio de los fenómenos naturales, típico de la ciencia moderna, sino con los valores que, más allá de lo epistémico, guían el desarrollo de nuestras capacidades tecnológicas. En este sentido, hemos revisado en la cuarta sección del trabajo dos problemáticas relacionadas con los medicamentos que van más allá de una evaluación técnica de su calidad, seguridad o eficacia. En primer término, hemos discutido los valores que guían la innovación en el campo farmacéutico. Allí pudo apreciarse que si bien los nuevos productos cumplen requisitos de seguridad y eficacia, la mayoría de ellos no representa un avance significativo respecto a los otros ya disponibles en el mercado. La eficacia se mide en términos absolutos (los nuevos son mejor que nada) y no relativos frente a los otros productos ya comercializados. ${ }^{9}$ Esto es con-

9 Ésta es una de las lagunas legales que las empresas utilizan para comercializar medicamentos me too. Cf. Angell, 2006 , p. 265 . 
sistente con la forma en la que la industria farmacéutica ha encarado la innovación en las últimas décadas, apostando seguro a la posibilidad de producir una droga me too para robar una porción de mercado a la competencia antes que invertir en $\mathrm{I}+\mathrm{D}$ para dar verdaderos saltos que beneficiarían mucho más ampliamente a la salud pública. En segundo lugar, hemos discutido la problemática del acceso, que se inscribe claramente en la tradición del medicamento como bien social, cuya distribución debe asegurarse para garantizar la salud pública. La iniciativa de medicamentos esenciales de la OMS toma nota tanto de la dificultad en el acceso por parte de gran parte de la población mundial como de la existencia de medicamentos me too, en los cuales la política de acceso debe ser posterior a menos que se compruebe una ventaja frente a otras opciones. La estrategia de la OMS de enfocar el tema de los medicamentos esenciales como un asunto de derechos humanos muestra claramente el origen social de las obligaciones respecto a estos artefactos tecnológicos (cf. Seuba, 2006). Por otra parte, puede indicar un camino a seguir en otras áreas como las de la innovación.

Las dos problemáticas sociales descriptas en torno a los medicamentos nos permiten referirnos a dos etapas diferentes en la formación de consensos en torno a riesgos sociales del desarrollo tecnológico. La OMS, trabajando constantemente desde 1975, ha logrado un amplio consenso acerca de la necesidad de garantizar el acceso universal a los medicamentos más importantes. Ello se ha plasmado en acciones efectivas como la creación de la Lista de Medicamentos Esenciales o la inclusión de estos medicamentos entre las provisiones básicas del derecho de acceso a la salud reconocido en tratados internacionales. Asimismo, OPS (2007) recopila una gran cantidad de experiencias fructíferas de aplicaciones de la lista de medicamentos esenciales en los países de América Latina. A diferencia de lo mencionado respecto del acceso, en las cuestiones referidas a la innovación en productos farmacéuticos no se ha logrado avanzar tanto. Suele afirmarse que la mejor manera de cambiar la dinámica imperante es reemplazando el sistema de patentes por uno de premios que otorgue grandes sumas a los descubridores de fármacos que lleven a saltos cualitativos, sin que esto derive en derechos exclusivos de comercialización para nadie (cf. Love, 2006, entre muchos otros). ${ }^{10}$ Sin embargo, no ha habido grandes avances concretos en esta línea, que implicaría modificar la estructura profunda del negocio farmacéutico. Por otra parte, si bien la OMS siempre ha abogado por la investigación en enfermedades desatendidas, esto no puede lograrse de modo efectivo sin un compromiso fuerte por parte de la industria, que lamentablemente no se ha registrado. Los grandes costos asociados al

10 De más está decir que el tema de la propiedad intelectual es central tanto en las cuestiones de acceso como de innovación. No lo tocamos porque es muy complejo y por cuestiones de espacio no podríamos hacerlo aquí en detalle. Los interesados pueden consultar Watal (2000); Attaran (2004) y el informe de la comisión sobre salud pública y propiedad intelectual de la OMS (2006). 
desarrollo de nuevos productos farmacéuticos obligan a pensar estrategias que van más allá de lo que los estados puedan cada uno por separado emprender. En este sentido una cooperación público-privada exitosa puede ser un instrumento fructífero para operacionalizar los valores sociales que puedan consensuarse en torno del desarrollo farmacéutico. Una experiencia interesante en este sentido es el lanzamiento de la Asociación de Artesunato y Mefloquina para el tratamiento de la malaria en el Brasil y el Sudeste Asiático, parte de una colaboración entre el laboratorio público Farmanguinhos (Fundación Oswaldo Cruz), la iniciativa para enfermedades desatendidas DNDI y un laboratorio privado en la India (cf. DNDI, 2008).

En última instancia, lo que este trabajo se propuso es elaborar una noción más amplia de regulación tecnológica. Usualmente entendida como legislación para evitar riesgos, suele restringirse a riesgos del tipo "material”. Las políticas destinadas a mitigar riesgos sociales suelen incorporarse bajo un paraguas distinto del regulatorio como los Ministerios de Ciencia, Salud o Desarrollo Social. Tras esto está la intención de tener organismos regulatorios de perfil netamente técnico con el fin de evitar la "politización de la ciencia". Lo que este discurso encubre es que los organismos regulatorios no están ajenos a las discusiones políticas y que muchas veces su juego político es mucho más sutil. Abraham y Reed (2002) atestiguan acerca de cómo una instancia que se presenta como netamente técnica como la ICH está atravesada por fuertes intereses políticos y económicos. Cabe aclarar que no estamos negando aquí la existencia de un espacio técnico de evaluación, ni pretendemos tampoco reducir los fundamentos de toda normativa técnica a una construcción social. Como señala Queraltó (2007), lo técnico remite siempre a lo social, aunque no se agote en ello. Es por esto que creemos que la regulación tecnológica debe pensarse teniendo en cuenta siempre el horizonte social en el cual tendrá aplicación, incluyendo explícitamente consideraciones acerca de las relaciones de lo técnico con lo político, económico y social. ${ }^{11}$ De este modo permitiremos abrir el debate público sobre muchas cuestiones que se presentan como meramente técnicas pero tienen fuertes supuestos sociales que si no pasarían inadvertidos.๑

Federico Vasen

Universidad Nacional de Quilmes,

Becario doctoral Consejo Nacional de Investigaciones Científicas y Técnicas (Conicet), Argentina.

federico.vasen@gmx.net

11 La noción de código técnico de Andrew Feenberg (1999) constituye una propuesta interesante para discutir la presencia de diversos valores sociales en el diseño tecnológico y evaluar cómo éstos terminan plasmados en afirmaciones y regulaciones que no ponen de manifiesto su origen social y se presentan como meramente técnicas. 


\section{ABSTRACT}

This paper deals with the interaction between technical regulations and social values in the pharmaceutical development. International regulation regarding medicines is analyzed departing from Tula Molina's distinction of material and social risk. First of all, I discuss the regulatory values of quality, safety and efficacy for medicines assessment. Secondly I focus on two subjects to illustrate the social risk associated with pharmaceuticals: the therapeutic value of innovations and the access to essential medicines. Finally, I propose that technical regulations should mention explicitly the social circumstances in which they will be implemented.

KeYwords • Technological risk. Technology Assessment. Technical regulation. Medicines. Pharmaceuticals. Philosophy of technology.

\section{REFERENGIAS BIBLIOGRÁFIGAS}

Abraham J. \& Reed, T. Progress, innovation and regulatory science in drug development. The politics of international standard setting. Social Studies of Science, 32, 3, p. 337-69, 2002.

ALTO COMISIONADO de la ONU para los Derechos Humanos. Pacto internacional de derechos económicos, sociales y culturales, 1966. Disponible en: <www.unhchr.ch/spanish/html/menu3/b/a_cescr_sp.htm〉. Acceso en: o7 jun. 2008.

. Observación general al art. 14: el derecho al disfrute del nivel de salud más alto posible. Comité de Derechos Económicos, Sociales y Culturales, 2000. Disponible en: <www.unhchr.ch/tbs/doc.nsf/8egc603f4,86

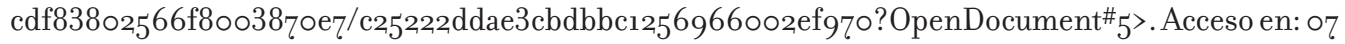
jun. 2008.

Angell, M. La verdad acerca de la industria farmacéutica. Cómo nos engaña y qué hacer al respecto. Bogotá: Norma, 2006.

Attaran, A. How do patents and economic policies affect access to essential medicines in developing countries. Health Affairs, 23, 3, p. 155-66, 2004.

Broncano, F. Mundos artificiales. Filosofía del cambio tecnológico. México: Paidós, 2000.

Diseños técnicos y capacidades prácticas. Una perspectiva modal en filosofía de la tecnología. Eidos, 6, p. $7^{8-121,2007 .}$

DNDI. Un nuevo producto resultado de un partenariado innovador, 2008. Disponible en: <www.dndi.org/ cms/public_html/insidearticleListing.asp? CategoryId = $166 \&$ SubCategoryId $=167 \&$ ArticleId $=475$ \&TemplateId=1>. Acceso en: ०७ nov. 2008.

Estébanez, M. E. Ciencia, tecnología y políticas sociales. Ciencia, Docencia y Tecnología, 17, 34, p. 24,-57, 2007 .

Feenberg, A. Questioning technology. New York: Routledge, 1999.

Garattini, S. Are me-too drugs justified? Journal of Nephrology, 10, 6, p. 283-92, 1997.

Green, G. Understanding NSAID: from aspirin to COX-2. Clinical Cornerstone, 3, 5, p. 50-60, 2001.

HogerzeIL, H. Essential medicines and human rights: what can they learn from each other? Bulletin of the World Health Organization, 84, 5, p. 371-2, 2006.

LEE, T. "Me too" products - friend or foe? New England Journal of Medicine, 35०, 3, p. 211-2, 2004.

Love, J. Drug development incentives to improve access to essential medicines. Bulletin of the World Health Organization, 84, 5, p. 4,08, 2006.

Molina, F. T. El contexto de implicación: capacidad tecnológica y valores sociales. Scientiae Studia , 4, 3, p. $47^{3-86,2006 .}$ 
Newton, P. et al. Murder by fake. The time for international action. British Journal of Medicine, 324, 7341, p. $800-1,2002$.

OMS. Revised procedure for updating WHO model list of essential drugs. WHO Executive Board rogth Meeting, Doc EB109/8, 2001. Disponible en: <www.who.int/gb/ebwha/pdf_files/EB1og/eeb1o98.pdf>. Acceso en: oๆ nov. 2008.

Salud pública, innovación y derechos de propiedad intelectual: informe de la Comisión de Derechos de Propiedad Intelectual, Innovación y Salud Pública. Ginebra, 2006. Disponible en:<www.who.int/ intellectualproperty/documents/thereport/SPPublicHealthReport.pdf >. Acceso en: ० ? nov. 2008.

The selection and use of essential medicine. WHO Technical Report Series, 946, Ginebra, 2007. Disponible en: <http://www.who.int/medicines/publications/essentialmeds_committeereports/ TRS946_EMedLib.pdf>. Acceso en: o7 jun. 2008.

OPS. Los medicamentos esenciales en las Américas: logros, dificultades y retos. Lima, 2007. Disponible en: <www.paho.org/spanish/ad/ths/ev/DocTecnico-Meds\%2oEsencs\%20en\%2olaRegión-2007.pdf>. Acceso en: 07 nov. 2008.

Parente, D. Encrucijadas de la técnica. Ensayos sobre tecnología, sociedad y valores. La Plata: Edulp, 2007.

Queraltó, R. Tecnología y desarrollo tecnológico. Un análisis filosófico para una evaluación axiológica. In: Parente, D. Encrucijadas de la técnica. Ensayos sobre tecnología, sociedad y valores. La Plata: Edulp, 2007. p. 81-124.

Quick, J. et al. Twenty-five years of essential medicines. Bulletin of the World Health Organization, 8o, 11, p. 913-4, 2002.

Quintanilla, M.A. Tecnología: un enfoque filosófico. Buenos Aires: Eudeba/Fundesco, 1988.

SeubA, X. A human rights approach to the WHO model list of essential medicines. Bulletin of the World Health Organization, 84, 5, p. 4.05-11, 2006.

Sismondo, S. Ghost management: how much of the medical literature is shaped behind the scenes by the pharmaceutical industry? Plos Medicine, 4, 9, p. 286, 2007. Disponible en: <dx.doi.org/10.1371/journal. pmed.004,0286>. Acceso en: 06 nov. 2008.

Trouiller, P. et al. Drugs for neglected diseases: a failure of the market and a public health failure. Tropical Medicine and International Health, 6, 11, p. 945-51, 2001.

UGALde, A. \& Homedes, N. From scientists to merchants. The transformation of the pharmaceutical industry and its impact on health. Societies without Borders, 1, p. 21-4,0, 2006a.

. Improving access to pharmaceuticals in Brazil and Argentina. Health Policy and Planning, 21, 2, p. 123-31, 2006b.

VilLA, S. et al. Orphan drug legislation: lessons for neglected tropical diseases. The International Journal of Health Planning and Management, 2008. Disponible en: 〈http://dx.doi.org/10.1002/hpm.930〉. Acceso en: 06 nov. 2008.

WATAL, J. Access to essential medicines in developing countries. Does WTO TRIPS Agreement hinder it? Science, Technology and Innovation Discussion. Center for International Development: Harvard University/Cambridge, 8, 2000. Disponible en: <http://www.hks.harvard.edu/cid/archive/biotech/papers/ discussion8.pdf >. Acceso en: 07 jun. 2008.

WaZANa, A. Physicians and the pharmaceutical industry. Is a gift ever just a gift? Jama, 283, 3, p. 373-80, 2000. 